Voix et Images

\title{
Post-modernisme et avant-garde au Canada, 1960-1984
}

\section{Caroline Bayard}

Volume 10, numéro 1, automne 1984

Littérature canadienne-anglaise

URI : https://id.erudit.org/iderudit/200456ar

DOI : https://doi.org/10.7202/200456ar

Aller au sommaire du numéro

Éditeur(s)

Université du Québec à Montréal

ISSN

0318-9201 (imprimé)

1705-933X (numérique)

Découvrir la revue

Citer cet article

Bayard, C. (1984). Post-modernisme et avant-garde au Canada, 1960-1984. Voix et Images, 10(1), 37-58. https://doi.org/10.7202/200456ar d'utilisation que vous pouvez consulter en ligne.

https://apropos.erudit.org/fr/usagers/politique-dutilisation/ 


\section{Post-modernisme et avant-garde au Canada, 1960 - 1984}

\section{par Caroline Bayard, Université McMaster}

Dans From There to Here deux critiques, Eli Mandel et Marshall McLuhan, sont désignés comme les prophètes involontaires (et peut-être inconscients) du post-modernisme au Canada. Le premier pour avoir décrit un raisonnement multiphasique, discontinu, englobant qui remplaçait à ses yeux la logique catégorisatrice et séquentielle de Platon; le deuxième pour avoir envisagé une autre approche critique face à une nouvelle littérature, face à des oeuvres ouvertes 1 .

Une approche qui n'essayerait pas d'imposer aux textes une structure, une logique, une grille raisonnée mais se tournerait vers eux avec une totale subjectivité. Une ouverture, comme le disait Mandel, à la phénoménologie ${ }^{2}$. Frank Davey conclut dans ce même texte ${ }^{3}$ que ce que ces deux hommes annonçaient c'était la fin d'une certaine ère, celle du modernisme et le commencement d'une nouvelle, électronique, décentralisée, post-électrique, post-autoritaire, en un mot post-moderne. La fin du modernisme, pour Davey, ce n'est pas seulement celle de la continuité, de la centralisation, de l'autorité mais c'est surtout l'écroulement d'un univers mythique où la stabilité, la reliogiosité et le sens esthétique de la fameuse "well-wrought urn" disparaissent au profit d'un monde imprévisible, fragmenté, non linéaire qui engage spectateur et lecteur dans une coopération anarchisante avec les éléments d'un univers où aucun élément ne domine l'autre4. Au modernisme d'Arthur James, Marshall Smith, James Reaney, Louis Dudek

1. Frank Davey, From There to Here, Erin, Press Porcepic, 1974, p. 18-19.

2. Roy Miki, "Talking West : an interview with Eli Mandel"' Line, no. 1, printemps 1983, p. 35.

3. Davey, du reste, nuance cette introduction en spécifiant que McLuhan était traditionnel dans ses goûts littéraires et prêtait fort peu attention aux auteurs contemporains alors que Mandel, par contre, avait su appliquer les théories mcluhanesques à la littérature et déceler ici une nouvelle écriture qui se déplacerait "inconsistently and incoherently amidst the fragments of personality and history, mixing space and time". Mandel est ici cité par Davey, opus cit., p. 19.

4. Ibid., p.20. 
et Phyllis Webb, à ceux qui voulaient contrôler à la fois leur univers et leur art, ont succédé les post-modernistes : bp Nichol, Victor Coleman, Daphne Marlatt, George Bowering, pour qui l'absence de contrôle implique la participation (condition désirable, normale, humaine). Au "masterwork" succèdent donc le désordre, l'intermittence. Aux mots d'ordre, l'irrégulier, le momentané. Le temps est au faux document (Michael Ondaatje avec The Collected Works of Billy the Kid), aux textes trouvés et présentés sans qualification d'aucune sorte, afin qu'ils établissent leur propre autorité, ou leur manque d'autorité (voir par exemple The Great Francisco Earthquake \& Fire de John Robert Colombo ou George Vancouver de George Bowering ou The Temptations of Big Bear de Rudy Wiebe)s.

Mais cette écriture que l'auteur de From There to Here décrit comme étant particulariste et phénoménologique ne bannit pas pour autant le mythologique. Différence capitale pourtant, ce mythologique n'est ni surimposé, ni externe au texte - Davey dirait "artifactual" - mais ancré dans l'environnement même du poète, dans l'océanique muraille du Pacifique de Daphne Marlatt, dans les psalmodies amérindiennes du bill bisset, dans l'hagiographie langagière de B.P. Nichol (les saints sont les mots, la vie des saints le langage).

Il serait vain de prétendre cerner en quelques pages le mouvement postmoderniste au Canada quand on sait que sa naissance remonte à un quart de siècle. Mais on peut essayer de dégager certaines de ses lignes de force. Géographiquement et chronologiquement parlant, certains faits s'imposent. On pourrait, en simplifiant quelque peu, avancer que tout s'est passé soit sur la Côte ouest, soit à Toronto6. Les expériences de nouvelles écritures ont commencé au début des années 60 , se sont développées au niveau de la performance dans les années 70 et précisées d'un point de vue théorique dans les années 80 .

Il y a cependant un problème sur lequel il convient ici de jeter quelque lumière : au Canada il n'y a pas eu d'avant-garde au cours des années 20 et 30. En fait si James Joyce, Gertrude Stein, les constructivistes et les futuristes se sont constitués les porte-parole de l'avant-garde entre les deux guerres (et un peu avant), au Canada personne ne semblait les connaître avant le début des années 60. Et si le Toronto Research Group semble "régurgiter"

5. Ibid., p. 22.

6. Il ne faut pas, toutefois, oublier que les Prairies ont fourni deux écrivains de taille: Rudy Wiebe et Robert Kroetsch. Robert Kroetsch est le plus représentatif dans l'univers de la narrativité post-moderne. Il se définit comme un écrivain subversif de l'Alberta. Voir Robert Kroetsch, "Death is a Happy ending : Dialogue in Thirteen Parts" dans Figures in a Ground, Diane Bessai et David Jackel, edit., Saskatoon, Western Producer Prairie Books, p. 207 et sq.

Le poète Andrew Suknaski aura un impact plus limité au cours des années 70 . Il participa à l'avant-garde poétique canadienne en brûlant ses poèmes sur des plages ou en les parachutant au-dessus de la Prairie, mais ses impulsions seront brèves et par définition auto-destructrices. 
une si énorme partie de l'histoire de l'écriture du vingtième siècle, explique bp Nichol, c'est en fait pour maîtriser et dépasser ce qui avait été occulté : "The way an amnesiac does when he tries to regain an awareness of his past 7". Il y a dans cet aveu de Nichol quelque chose à la fois de naïf et de surprenant. Certes, on comprend que le Canada de l'entre-deux-guerres, celui d'A.J.M. Smith et de Frank Scott, n'ait pas su ou pas voulu prendre acte de cet éclatement de l'écriture. C'est que cet éclatement s'était surtout produit en Europe (Dada en Suisse et en Allemagne, le surréalisme en France, le futurisme en Italie). Par ailleurs Joyce et Stein avaient fait leur part sinon culturellement du moins linguistiquement dans l'univers anglosaxon. Mais ni la colonie d'alors (l'Ontario), ni finalement la mère patrie (l'Angleterre) n'avaient été concrètement touchées par ces mouvements littéraires.

Le modernisme, lui, avait eu ses adeptes et avait connu d'intéressants développements dans le Dominion du Canada. Mais modernisme et éclatement du langage sont des pôles opposés. Et Thomas Stearns Eliot aura finalement été bien mieux entendu de son temps que l'Irlandais qui allait déchaîner une tempête structurale, sémiotique et sémantique dans cette langue qui n'était pas vraiment la sienne. Ce serait un contre-sens que d'interpréter le modernisme de Wystan Hugh Auden et de Thomas Stearns Eliot (et l'impact qu'ils ont pu avoir de ce côté-ci de l'Atlantique) comme étant, même de loin, apparenté à ce qu'il est convenu d'appeler, dans la francophonie, la modernité. En fait, quand les francophones parlent de modernité (on pense ici à l'intelligentsia de $\mathrm{Tel}$ Quel ou à l'équipe qui gravite au Québec autour de la Nouvelle Barre du jour et des Herbes rouges), les anglophones, eux, utilisent l'expression post-modernisme. Le règne du signifiant, de ce que d'aucuns appellent le côté gauche du triangle sémiotique (le Zeichen-Sinn au détriment du Bedentung-Sinn) coöncide avec la montée du post--modernisme en Amérique et ailleurs8. Le signifiant est devenu la référence absolue, la monade toute-puissante sur laquelle repose le système du sens (Jean Baudrillard9 et Julia Kristeva10 ont abondamment commenté ces développements).

Comment se met-on à parler d'une avant-garde canadienne dans les années 60 ? Et, si l'on tient compte de l'inévitable caractère artificiel de telles définitions, comment ce schéma fonctionne-t-il dans les années qui suivent? Il y a certainement eu, dès le début de cette décennie-là, des "expérimentations". Le terme a une connotation trop scientifique pour fonc-

7. “Interview with bp Nichol, Capilano Review, no. 8-9, automne 1975-1976, p. 333.

8. Voir là-dessus Charles Russel, "The avant-garde in the post modern era", ch. 2 in The Avant-Garde Today, Urbana, Univ. of Illinois Press, 1982, p. 6-15. H. Hassan, Paracriticisms, Urbana, Univ. of Illinois Press, 1975 et Jean-François Lyotard, Dérives a partir de Marx et de Freud, Paris, Union Générale d'éditeurs, 1973, p. 243-247.

9. Jean Baudrillard, Pour une critique de l'économie politique du signe, Paris, Gallimard, 1972, p. 100-107. Voir en particulier ses remarques sur le fétichisme du signifiant et la passion du code.

10. Julia Kristeva, Révolution du langage poétique, Paris, Seuil, 1974, p. 29. 
tionner adéquatement dans un contexte littéraire et pourtant il a eu une vie fort longue et curieuse. Bowering Il parle d'avant-garde, d'expérimentation et de tradition "minoritaire", Nichol 12 de nouvelle écriture qui est aussi une recherche, Marlatt de corps et d'immédiateté13. Quant au terme même d'avant-garde, il fait problème. D'abord parce qu'il a été employé régulièrement depuis un siècle et demi en référence à des manifestations textuelles, visuelles et dramatiques extrêmement diverses ${ }^{14}$. Ensuite parce que bien des poètes contemporains canadiens l'emploient avec une remarquable (et troublante) facilité sans pour autant préciser ce qu'ils entendent par ce fourre-tout commode qui est au menu de la textualité ce que la macédoine a dû être aux cuisiniers parisiens vers 1899.

Les frontières complexes entre les avant-gardes de ce siècle et le postmodernisme ont été décrites de manière prudente par le critique américain Charles Russell. Ces deux mouvements, nous dit-il, ont régulièrement focalisé leurs recherches sur les matériaux du texte et sur leurs structures. Pourtant les deux écoles s'opposent dans la mesure même où les stratégies innovatrices du post-modernisme (voir John Barth, Alain Robbe-Grillet, continue-t-il) ont toujours insisté sur la nature fictive, fabriquée de toute expérience encodée dans le langage alors que l'avant-garde, elle, préfère échapper à l'encapsulation, dépasser l'hermétisme et se diriger à la fois vers ce qu'il nomme une texture souple, subjective, nouvelle et vers les mécanismes complexes du discours collectif 15. Mais la distinction du critique de l'Illinois University Press résiste mal aux innombrables ambiguïtés non résolues. Comment définir et analyser l'écriture de post-modernistes tels Pynchon, Fuentes et Coover, paradoxalement déterminés à lier autarcie textuelle et histoire/mythe collectif? Le flou terminologique demeure total et bien malin ou bien imprudent sera celui qui tentera de l'effacer au profit de schèmes ou de catégories aussi souples fonctionnellement que pragmatiquement convaincants, du moins pour ceux qui essayent de cerner les limites du problème au Canada.

11. George Bowering, Out-Post/Avant-Postes, Caroline Bayard et Jack David, édit., Erin; Press Porcepic, 1978, p. 79.

12. Ibid., p. 28.

13. "Given this body an interview with Daphne Marlatt", Open Letter, printemps 1979, p. 33: "Avant-garde is simply writing as close as you can to what you are actually experiencing at a given point"'.

14. René Lourau, "L'auto-dissolution des avant-gardes", Paris, Ed. Galilée, 1981. Voir aussi Jeff Berner, édit. Astronauts of inner-space: an international collection of avantgarde activity, San Francisco; Stolen Paper Review editions, 1966. Robert Estivals, "Schéma pour l'Avant-garde", Revue de l'Université de Bruxelles, No 3-4, 1976, pp. 242-291. Miklos Szabolesi, "Avant-garde, Néo-avant-garde, Modernism, Questions and Suggestions", New Literary History, vol. III, 1971-1972, pp. 49-70. Philippe Sollers et Jacques Henric, "Pour une avant-garde révolutionnaire", Tel Quel, hiver 1970, no 40, p. 58-66. George Rochberg, "The avant-garde and the aesthetics of survival", New Literary History, vol. III, 1971-1972, pp. 71-92.

15. Russell, p. 9. 
Il demeure cependant que la Côte ouest du début des années $60 \mathrm{~s}$ 'est révélée un espace unique et privilégié pour le développement de l'expérimentation poétique. En fait c'est là que tout a commencé, autour de la revue Tish (1961 - 1969). George Bowering, Frank Davey, Frederic Wah y collaboraient régulièrement et parfois Lionel Kearns, l'inventeur de la théorie du "stacked verse", sur laquelle je reviendrai plus loin. Curieusement, Daphne Marlatt, dont les textes explicitent les théories sur le souffle, l'énergie, le vers, la marge et la ponctuation et offrent une parenté évidente avec les théories élaborées par les collaborateurs de la revue Tish, n'a jamais participé aux travaux du groupe. Curieusement aussi c'est elle qui, sans se joindre à aucun mouvement ni école, intéressera le plus fortement les femmes qui pratiqueront la nouvelle écriture au Québec. Le lien traverse plusieurs géographies, spatiales, politiques, génétiques, biologiques. Tish a eu des rapports fréquents et continus avec les poètes américains des deux côtes. Un des mentors de Tish, Warren Tallman, était Américain. Il enseignait à UBC. Charles Olson, Robert Creeley et Robert Duncan font partie du champ référentiel de la revue. Tish a repris et amplifié deux principes olsoniens. Tout d'abord l'écriture doit son allégeance à la voix et non à l'intellect, c'est-à-dire au souffle, au son, à la pulsion phonique ${ }^{16}$. Le poème, dira Olson, est " energy-gift, energy-construct, energy-discharge ${ }^{17}$ ". Deuxièmement le texte est image, contact visuel. La transcription du souffle dans les vers, de la syllabe sur la ligne horizontale a longtemps préoccupé Lionel Kearns. Comment projeter l'inflexion de la voix sur une mesure graphique et métrique, comment indiquer les accents toniques? Son objectif sera d'établir un système de transcription dans lequel la mise en page refléterait totalement la phonie, dans lequel rythme et temps de visualisation, image, seraient rigoureusement isomorphiques ${ }^{18}$. Cela donne les résultats suivants:

\title{
Situation
}

\author{
Coffee beans \\ in the morning sun,
}

16. William Carlos Williams, "not in ideas but in things" et l'injonction de Charles Olson, "man \& external reality are so involved with one another, that, for man's purposes they had better be taken as one", cité par Warren Tallman dans Tish, no 3, novembre 1961, p. 13. Tallman ajoute du reste son propre commentaire: "the human house is note here to be so many bleak walls from which the intellect must seek some exit into some elsewhere before death collapses the house into ruins." Ibid.

17. Voir Lionel Kearns, "Stacked Verse", Delta, no. 19, 1962, p. 15-20. Voir aussi du même auteur "The Rationale of Stacked Verse", Open Letter, printemps 1982, Se série, no. 2, p. 21-38.

18. Lionel Kearns, "Situation", in "The Rationale of Stacked Verse", Open Letter, printemps, 1982, Sème série, no. 2, p. 35. 
42 VOIX ET IMAGES, VOL. $X$, NO 1 (AUTOMNE 84)

\author{
A giant hog \\ asleep \\ between the trays, \\ And once \\ Alfonso \\ climbed the hill \\ And talked \\ and stayed. \\ And in the afternoon \\ we sat in the finca \\ sipping coco-nuts \\ and rum \\ With faint guapango rhythms \\ drifting up \\ from some marimba band \\ down in the village, \\ And later on \\ strolled down the hill \\ ourselves. \\ Small-talk \\ at the stall \\ of Mama Lupita, \\ Funny stories \\ over at \\ the cantina, \\ A ride \\ to Catamaco, \\ reflections \\ on the lake.
}


Les lignes verticales du 'stacked-verse' tenteront de contrôler le débit phonique du poème et de fusionner le visuel et le phonique18a. Ce système ne sera pas repris par d'autres poètes et Kearns lui-même l'abandonnera après quelques années. Mais l'impulsion isomorphique son-sens, sonimage, - rappelons ici l'injonction de Charles Olson: "A line must be as white, as warped as wood is" - sera récupérée par une autre avant-garde, celle du concrétisme.

Souffle. Phonie. Rime. Tous les poètes de Tish et même ceux qui partagent les préoccupations de Tish comme Marlatt reviennent là-dessus 19.

Bien qu'elle ait été la plus périphérique, "on the edge" dira Bowering20, la moins impliquée dans la politique textuelle de Tish, Marlatt n'en demeure pas moins la formulatrice la plus intéressante de ces préoccupations au sujet du souffle, de la matérialité de l'énergie, du corps nécessairement impliqué dans l'écriture et de la corporalité de cette dernière21. Quand on songe aux dénominateurs constants de Charles Olson, l'auteur de Projective Verse, à sa concentration sur la "proprioception" - la physiologie des stimuli qui proviennent de l'intérieur du corps 22 l'on se rend compte que c'est Marlatt (plus limpidement que George Bowering ou Frank Davey, poètes et théoriciens de Tish) qui aura saisi cette fusion corps-sens, soncorps, corps-son. Pour elle tout mot est un corps, "Any word is a physical body" (Selected Poems, p. 16) et elle intitulera le long dialogue d'Open Letter: "Étant donné la réalité de ce corps"' "Given this body", Open let-

18a Une préoccupation du groupe Tish (que les concrétistes de tout bord reprendront) sera l'utilisation de l'espace vide, du blanc typographique, utilisé par eux comme silence et par leurs successeurs comme stratégie textuelle. Voir William S. Choy "Space and its relation to poetry", Delta, no 19, octobre 1962, p. 21-22. Voir aussi le commentaire que fait bp Nichol sept ans plus tard sur l'utilisation de ce fameux espace blanc chez bill bissett : "This (the blank) sets up areas of stress in which the brain strains to make the imaginative jump and fill in the gaps that bissett has left". "The typogeography of bill bissett", in We sleep inside each other, All Toronto, Ganglia Press, 1966, sans pagination.

19. Entrevue dans Open Letter, printemps 1979, p. 85.

20. Ibid., p. 35.

21. "Plus (...) la volonté propre de la matière, l'autonomie irréductible du langage, " Ibid., p. 59-60.

Le langage a une présence, des insistances et des réseaux de liens qui lui sont propres, et ce sont ceux-ci dont nous devons sans arrêt tenir compte. Comme le sculpteur qui travaille avec la pierre, la pierre est intraitable, elle a son grain, et sa manière à elle de se briser, s'il opère maladroitement elle se cassera exactement comme il ne voulait pas qu'elle se casse. "Given this body", p. 59.

22. Voir l'introduction de Fred Wah aux Selected Writings of Daphne Marlatt, Vancouver, Talon Book, 1980, p. 16, 17, 19. 
ter, printemps 1979, p. 32 - 88)23. L'un des critiques de Marlatt liera cette sensualité du langage à un corps à corps de la géographie et de la syntaxe, de l'histoire et de la mise en page, du politique et du typographique. Steveston (1974), l'un de ses volumes de poésie les mieux connus, retrace la vie de pêcheurs canadiens d'origine japonaise, dans un petit village isolé et battu par les vents de la Côte ouest. D'après Fred Wah ce sont précisément ces audacieux mouvements de la mise en page, ces blancs, ces ruptures qui réflètent la sémantique de ce mouvement de masse humaine à travers l'océan, le typographique et l'historique se fusionnant jusqu'à l'embouchure du Fraser, jusqu'à la bouche du poète 24 . Voyons plutôt la respiration que donne Marlatt à cette ville et aux visages qui la peuplent :

Shadowy, this piratical emblem af another ear. Boomtown. Dream of seizing silver wealth that swims, \& fixing it in solid ground, land, home. A mis-reading of the river's push. Now Moncton Street walks a straight line that begins \& ends. Never a $24 \mathrm{a}$

Et la phrase s'interrompt ici.

C'est ce qu'Altieri, dans ses réflexions sur la poétique post-moderniste, appellerait la fusion du lieu et du langage25, ce que Douglas Barbour26 nomme une poétique phénoménologique. C'est la démarche qui accepte l'exhortation d'Olson à s'éloigner des généralisations pour plonger dans le corps de la matière ("language is a prime of the matter27"). Ce sont des textes qui illustrent l'exigence de Merleau-Ponty qui veut que nous soyons les témoins à chaque instant du miracle d'expériences conjointes, nul ne sachant mieux que nous comment ce miracle se dessine puisque nous sommes nous-mêmes une trame et une texture de relations et de liens 28 . La métaphore la plus percutante que Marlatt donnera de ce choix dans ses poèmes est celle de l'oiseau qui survole un cours d'eau. On pourrait

23. "I am reallgiven to the sensual. I really delight in that... language is leafing our, it's everything that is growing, that is organism, that is body. It's a body, I love that phrase, the body of language. And I am trying to realize its full sensory nature as much as possible. We live in the world, that's my basic assumption. I want to learn everything I can about what it is to live in this world, to be mortal, which 1 take to be in the body". ("Given this body", p. 60) Cette conception de la matérialité du corps a été du reste partagée par toute la génération de Tish. Voir Frank Davey, "Starting at our skins", ibid., p. 89.

24. Voir l'introduction de Fred Wah à opus cit., p. 19.

24a Daphne Marlatt, opus cit., p. 19

25. Charles Altieri, "From Symbolist Thought to Immanence : the Ground of Post-Modern American Poetics", Boundary 2, $1: 3$, printemps 1973, p. 608.

26. Douglas Barbour, "Marlatt's Stevenston" in Figures in a Ground, Diane Bessai \& David Jackel édit., Saskatoon, Western Producer Prairie Books, 1978, p. 178.

27. Charles Olson, Human Universe \& Other Essays, New York, Grove Press, 1967, p. 4.

28. Maurice Merleau-Ponty, Phénoménologie de la perception, Paris, Gallimard, 1976, p. 85. 
observer, dit-elle, cet oiseau de la rive opposée, mais là n'est pas ce qui m'intéresse. Mon seul désir est de nous faire entrer dans le corps de cet oiseau, en plein vol, et de déchirer l'air avec lui. Et parce que nous sommes en plein vol, parce que nous sommes son corps, celui qui lit, quel qu'il soit, fait corps avec l'oiseau, avec moi, avec nous, avec l'air 29 .

La même démarche phénoménologique se retrouve chez bill bissett, un autre poète de la Côte ouest. Certains de ses critiques ont fait référence à l'énergie libidinale de son écriture, réseau anarchique de pulsions contradictoires, souvent violentes, flot de ruptures et d'excès dira Steve McCaffery30. Ce dernier situe la problématique de cette écriture dans un cadre familier aux lecteurs francophones, celui exposé par Deleuze et Guattari dans l'AntiOedipe, par les travaux d'Antonin Artaud et Georges Bataille sur l'économie du désir31 et la réflexion de François Fourquet sur "Nietzche libidinal32". Mais la poétique de bissett se situe en un lieu textuel différent de celui de Marlatt. Il ne s'agit plus seulement ici d'instantanés vécus, d'immédiatetés, de plongées dans la matière, mais de disjonctions, de brouillages (pistes / sons / codes / termes / typographies). Ce que McCaffery appelle une expérience de l'anti-grammaire, de l'anti-langue, un mode de désir schizophrénique opérant à travers et malgré la langue33.

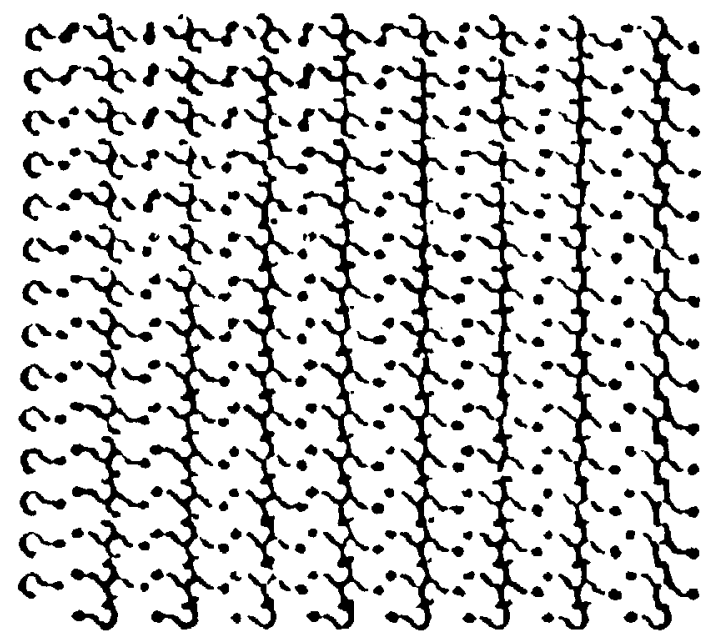

Image beings (Blew Ointement, 1975)

29. Voir "Given this body", Open Letter, printemps 1975, The Selected Writings of Daphne Marlatt, p. 14.

30. "bill bisset: a Writing Outside Writing" Open Letter, 3e série, no.9, automne 1978, p. 17.

31. Ibid., p. 21, 22, 23.

32. François Fourquet, "Nietzche libidinal", Sémiotext(e), III, 1, 1978, p. 11.

33. "Writing Outside Writing", op. cit., p. 2. 


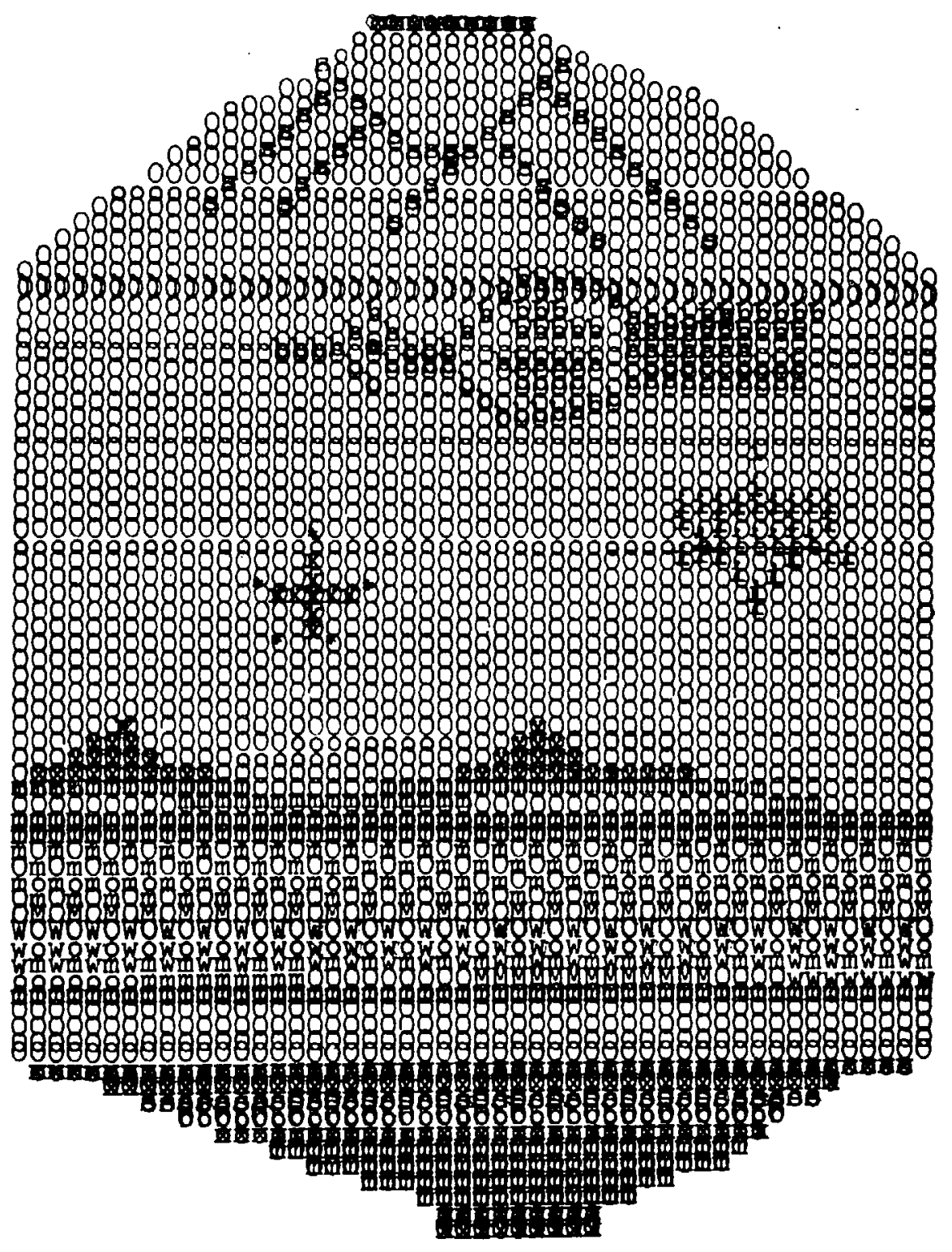

Soul Arrow - bill bissett

Image beings (Blew Ointment, 1975) 
Si l'on examine Soul Arrow ou Image beings on est en droit de se poser un certain nombre de questions, la première et la plus fondamentale étant de savoir ce qui reste de cette masse d'encre indifférenciée qui semble être la marque emblématique de bill bissett. Il serait injuste de prétendre que la "révolution du langage" qu'il annonce se limite à de telles manifestations 34 . En fait, quand bissett plaide pour l'anarchie orthographique et sémantique, il nous exhorte aussi à une libération individuelle et collective, subjective et politique. En nous demandant de rejeter notre allégeance " to the ruling class of meaning" ( $\mathrm{sic})^{35}$, il nous incite aussi à nous ouvrir à l'énergie des mots, à leurs impulsions, à leurs vibrations ${ }^{36}$. Il est conscient du reste du caractère paradoxal de son exhortation: exiger qu'on abandonne toutes règles peut devenir inéluctablement une autre règle:

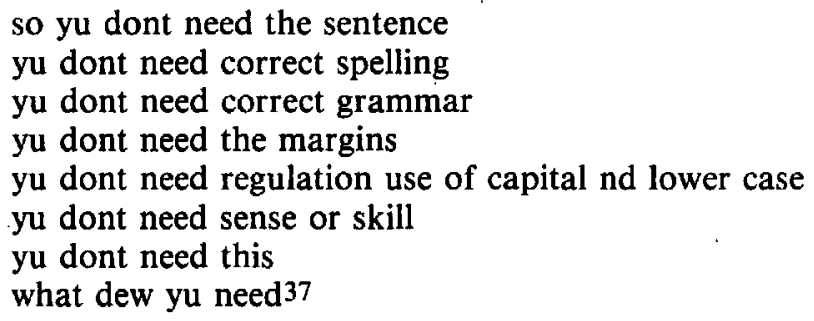

et bissett de conclure : "but $\mathrm{i}$ aint delining/defyin or generalizing/just frget all th rules/suspend it 38 ".

Nous pouvons tous suspendre, disséminer, changer, alterner ou recréer le(s) sens. Le sens est aussi éclaté qu'il est total et unifié. Une des tendances les plus révélatrices, les plus explicites dans le champ conceptuel de bissett est sa croyance en la dissémination du sens et simultanément en son unité. Une sorte de paganisme absolutiste : l'esprit est un et partout, le sens est tout et en tout et aussi ce que nous voulons qu'il soit. Une des ses exhortations récurrentes est: "let th pomes speak fr themselves", "reject th imperialism of semantiks". Il s'agit d'un programme difficile pour les tenants d'un rationalisme intégral. L'anarchie linguistique de bissett est inséparable de sa vision politique, son sens de la subversion autant poétique qu'institutionnel. Les textes les plus explicites à cet égard sont nobody owns th Earth (1971), Lost Angel Mining Company (1969), Pass th Food, Release th Spirit Book, (1973), Ice (1972). Len Early replace cette subversion dans le contexte plus large de la contre-culture américaine des années 60 , des textes de Théodore Roszak (The Making of the Counter-Culture) et de R.D. Laing39.

34. On devrait noter, incidemment, le jeu de mots fréquent auquel il se prête, "language revolution" devenant aussi "lung wage revolution" ou comment débarrasser nos poumons du poids et des carcans qui les oppriment.

35. What Fuckan Theory, Toronto, Cronk Press, 1971, sans pagination.

36. Pass th Food, Vancouver, Talon Books, 1973, sans pagination.

37. What Fuckan Theroy, sans pagination.

38. Ibid.

39. Len Early, "bill bissett: Politics, poetics \& vision." Essays in Canadian Writing, automne 1976, no 4, p. 4, 22. 
Il y a deux aspects de l'écriture de bissett qui attireront l'attention (dans un double jeu d'opposition/identification) des poètes québécois. Le premier concerne les collages-montages, un choix que tous les tenants de la jeune écriture, sous tous les cieux, ont pratiqué entre 1967 et 1975. Au Québec on pourrait citer Denis Vanier, Josée Yvon, Paul Chamberland40, Raoul Duguay, Patrick Straram. Une relecture de Mainmise confirmerait cette dernière affirmation. Sur la Côte ouest il faudrait aller du côté de Blew Ointment (la revue publiée par bissett, pas la maison d'édition) pour retracer ces mêmes influences. Le montage de découpures de journaux, de photos, de fragments de poèmes ou d'alphabet, qu'il ait été produit au Canada, au Québec, au Brésil ou en Allemagne, révèle, en fin de compte, une même intention.

C'est un héritage de Dada qui se veut aussi texte ouvert, multiforme, polysémique, texte incitant le lecteur à la participation en lui suggérant des niveaux de lecture multiples (de gauche à droite, de droite à gauche, de bas en haut, en diagonale, etc. $)^{41}$. Les collages de bill bissett ont une portée politique indéniable 42 . Les liens entre la grande industries et la vente des armes, les valeurs bourgeoises, les politiciens d'Ottawa, Nixon, Reagan et la bombe atomique en prennent généreusement pour leur compte. C'est un genre qu'il a quand même plus volontiers pratiqué dans les années 60 et 70 que dans la décennie suivante.

Le deuxième aspect a trait à la spiritualité, à ce que l'on pourrait nommer le pouvoir métaphysique du son. Des commentaires semblables ont été faits au Québec par Raoul Duguay et Paul Chamberland qui se sont ainsi démarqués de leurs anciens compagnons marxistes ou des formalistes de $l a$ Nouvelle Barre du jour. De tels commentaires nous ramènent du reste à la contre-culture et à R.D. Laing, mais ils dépassent également ce cadre et se retrouvent dans le champ plus global, moins historiquement déterminé par le mysticisme. Ce qu'il y a de particulièrement original chez bissett, c'est la volonté de lier son et souffle - il dira " tongue \& breath are the way fr speaking tongues to pass 43 " - au souffle du Grand Esprit, des mythes indiens, le shaman n'étant ici qu'une voie de passage vers la vaste totalité du souffle suprême. Voilà pourquoi bissett, dans ses lectures publiques, psalmodie, scande et danse ses poèmes. Plusieurs critiques ont noté l'influence amérindienne de ces pratiques 44 .

40. Voir en particulier "Vers le grand changement", Mainmise, octobre 1974, p. 21.

41. Sur le collage en tant que forme littéraire et visuelle voir Gérald F. Brommer, The Art of Collage, Worcester, Mass. Davis Publications, 1978 et Herta Wescher, Collage, New York, Harry N. Abrams, 1968.

42. Voir Len Early là-dessus, op. cit., p. 8.

43. Pass th Food, sans pagination.

44. Voir Len Early, p. 11. Mentionnons aussi le commentaire de Frank Davey, "These chants are based on West Coast material", ibid., p. 11. 
La répétition qui caractérise les psalmodies de bissett a de fortes connotations rituelles et sacramentales. Len Early souligne à quel point une grande partie de sa poésie orale équivaut à une utilisation religieuse du langage et est étroitement liée à une vision du sacré qui transparaît dans des volumes comme Polar Bear Hunt (1972) et Th High Green Hill (1972)45. De telles ouvres ont été parfois dénommées des " tapisseries sonores" et en fait on décèle ici une parenté avec certains de ses textes visuels : structures et échafaudages typographiques qui veulent échapper au sens et qui tissent une trame visuelle. Cette volonté a du reste ses limites puisque bissett choisit aussi la voie de l'isomorphisme cher aux concrétistes : sens et typographie ne font plus alors qu'un. Cette fusion du sémantique et du visuel se rencontre fréquemment et utilise avec humour, quand il ne s'agit pas de malicieuses provocations, la sexualité et le désir. Voir par exemple le fameux "69" de A wake in the Red Desert.

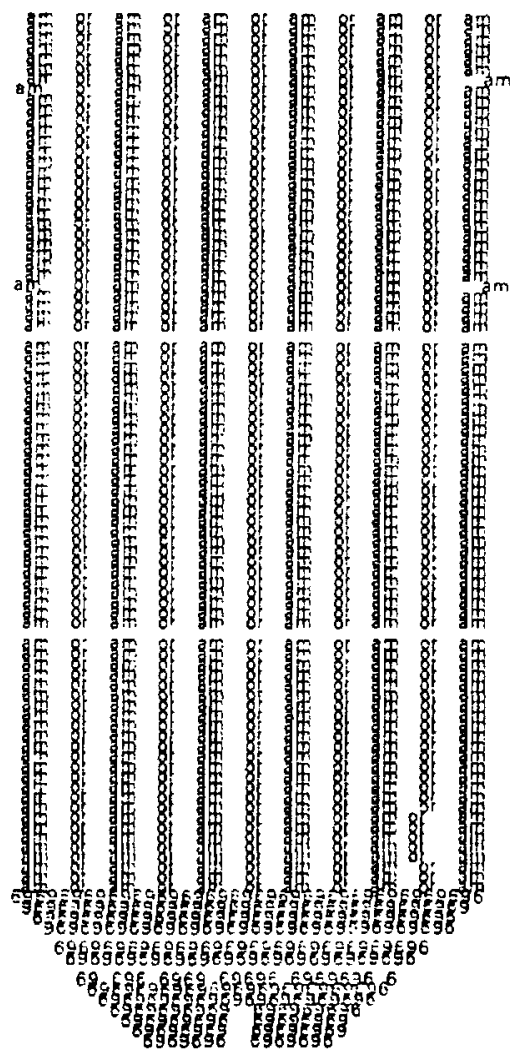

45. Ibid., p. 14. 
Une dernière remarque s'impose au sujet de bissett: l'utilisation systématique de la machine à écrire, marque de commerce de son écriture, le distingue des autres post-modernistes au Canada. Étant donné sa méfiance chronique à l'égard de la technologie, de l'industrialisation, des institutions politiques, un tel choix peut surprendre (pensons aussi à l'amour de la calligraphie partagé par d'autres post-modernistes, Paul Chamberland, Raoul Duguay). Mais cette prédilection est aussi un choix politique. Pour bissett la machine à écrire représente l'accès démocratique et universel au monde de l'imprimerie. Chaque individu peut ainsi créer sa propre maison d'édition et ronéotyper des feuilles tapées à la machine. On peut songer aussi à des raisons formelles et stylistiques : un tel outil de production permet une utilisation consciente et contrôlée de la page. Et une conscience aiguë de l'espace de la page est l'un des objectifs précis de bissett.

the typewritr is amazing how it

meens (that)

yu can place th words on th page wher

th moovment rhythm sound change happins use th space inbtween th words 47

Placés à côté des feuilles tachées d'encre de bissett, peu d'écrits pourraient présenter des contrastes plus saisissants que ceux du Toronto Research Group. Composé de Steve McCaffery et de bp Nichol, le Toronto Research Group publie le gros de ses réflexions théoriques dans Open Letter, l'équivalent canadien de la Barre du jour48. On chercherait en vain dans le Fuckan Theory de bissett quelque référence savante aux grands penseurs des derniers siècles. Le terme "théorie" est employé, dans ses textes, avec une intention parodique, et si théorie il y a, ce serait un principe d'antithéorie. Surgit donc le TRG, dix ans après que Bissett ait commencé à publier ses poèmes 49 . Son cadre de référence est explicitement ancré dans le post-modernisme et inclut non seulement les concrétistes brésiliens, allemands et français, mais aussi les explorations textuelles de Gertrude Stein, James Joyce et des poètes sémiotistes américains (Bruce Andrews, Ray di Palma, Barbara Barracha, Ron Silliman50). Il semble également que le TRG ait fréquenté les écrits de Merleau-Ponty, Ponge, Foucault et Derrida.

47. Fuckan Theory, op. cit., sans pagination.

48. Voir l'article de Barbara Godard "The Avant-garde in Canada : Open Letter and la Barre du jour, Ellipse, nos 23-24, 1979, p. 98-113 et celui de Caroline Bayard, "Deux pièces difficiles pour une même main", ibid., p. 156-173.

49. Bissett émerge grâce à Tish au début des années 60 , puis lance sa propre revue Blew Ointment en 1963. En 1963 le Toronto Research Group commence à publier ses textes dans Open Letter et n'a pas cessé depuis.

50. Pour plus de commentaires sur ces derniers, voir "The Politics of the Referent", Open Letter, 3e série, no 9, été 1977, p. 61-77. 
L'association Nichol-McCaffery a le troublant avantage de donner des textes où ni l'un ni l'autre des participants n'est identifié. Cette collaboration ne se veut pas un dialogue mais une recherche de nouveaux modes de lecture, d'interprétation et d'écriture. De fait le manifeste comme forme d'écriture ne les a pas satisfaits; ils l'abandonnent donc rapidement 51 . Les "rapports" du TRG sont ainsi nés, alternativement longs ou brefs, bourrés de références américaines et françaises, laconiques, télégraphiques, parfois hermétiques, pratiquant souvent l'art du raccourci, du percutant, de l'implicite mais déterminés à analyser la demeure dans laquelle nous vivons - le langage - et sûrs de pouvoir saisir les modifications introduites par ce qu'ils appellent - paradoxe curieux - la tradition avant-gardiste52.

La fonction de la théorie est capitale pour le TRG : elle est la seule entité qui puisse nous aider à enjamber les écarts, à comprendre les éléments hétérogènes qui peuplent nos maisons et nos corps (onomatopées, cris primals, manipulations électroniques, styles édouardiens). Après tout, nos maisons de Toronto, disent-ils, ont été construites à une autre époque tout en fonctionnant aujourd'hui dans un univers hautement technologique53. Les prémisses épistémologiques du TRG sont clairement articulées autour de concepts ontologiques post-hegeliens et husserliens. Le sens n'est pas une essence, n'a pas d'existence propre et ne peut être dérivé que de l'expérience, de la perception, de la réception d'un objet. Tout en étant conscients des bouleversements et des déplacements qui ont affecté la littérature et la linguistique depuis cent ans - particulièrement la fusion que d'aucuns ont voulu pratiquer entre le signifiant et le signifié: "so that they be brought together into a visual instantiation of an object-event 54 " - ils proposent une démystification du référent-fétiche qui déguise et exclut le processus de travail du texte. Leur volonté est de transformer la signification en un processus centripète: l'énergie au lieu de couler vers l'extérieur, vers un concept ou un objet situé hors du champ textuel, se dirigera au contraire vers le signe-graphème. Au lieu de consommer le texte, au lieu d'absorber le sens, ce produit-fétiche, nous lecteurs pourrions introduire $d u$ sens à l'intérieur de ce signe précédemment vidé. Le lecteur devient producteur; le texte, un processus de transformation-production; le signe, une configuration relationnelle. Les textes post-modernistes n'opèrent pas exclusivement à travers des signes d'où le sens a été évacué mais ils manifestent une préférence en ce sens.

51. Voir le "Manifesto II" qui rapporte la perte du "Manifesto I", Open Letter, 2e série, no 4, printemps 1973, p. 75.

52. "TRG Report 2: Narrative (part 5)", “Manifesto as Interlude”, Open Letter, 2e série, no 9 , automne 1974 , p. 73.

53. Du reste pour le TRG il n'y a pas de "mouvement" dans le sens d'une histoire littéraire linéaire mais "an interconnectedness of concerns which goes back to Saussure, the Russian Formalists, Barthes, Lacan and Derrida". "The Politics of the Referent", opus cit., p. $61-77$.

54. Voir l'abondante documentation qu'ils fournissent là-dessus, de George Herbert et Apollinaire jusqu'aux concrétistes contemporains: "TRG Report 2: Narrative Part I ", Open Letter, 2e série, no 6, automne 1973, p. 117. 
Lorsqu'on analyse les écrits du TRG qui s'échelonnent sur une période de dix ans, on se rend compte que leurs auteurs voudraient pré-vider, évacuer toute signification du signe mais se trouvent pris au piège de contradictions diverses. Finalement, ils reconnaissent qu'il est impossible d'évacuer totalement les décharges mnémoniques hors du signe55. L'effort a cependant, à leurs yeux, une valeur pragmatique : ils déconstruisent une certaine structure de pouvoir, celle de la vieille hiérarchie écrivainproducteur/mots producteurs de sens/lecteur-récepteur-consommateur de mots. Déconstruction qui démet l'auteur de son droit divin et révèle un nouveau processus au bout duquel le texte est toujours lacunaire56. Jusqu'à quand demeurera-t-il tel? Le TRG pose ici l'alternative suivante:

1) le lecteur acceptera ce degré zéro, cette vacuité sémantique57, comme densité pure, événement;

2) le lecteur choisira d'augmenter et de compléter le texte, se transformant ainsi en logothérapeute ou logotecte58.

Le signe évacué, le* "zérocipher" est ce qui fait problème dans l'herméneutique du TRG. En fait il est défini ici comme un signe privé de sa fonction, un signe à observer et à percevoir en tant que signe. C'est une évacuation, une privation qui pose de sérieux problèmes ontologiques et dont les effets ont été analysés par Derrida dans l'Écriture et la Différence: en effaçant la différence radicale entre $s a$ et $s e ́$, "c'est le concept de $s a$ luimême qu'on est forcé d'abandonner comme concept métaphysique 59". Le concept même de signe ne peut "dépasser cette opposition du sensible et de l'intelligible. Il est déterminé par cette opposition de part en part et à travers la totalité de son histoire. (...) nous ne pouvons donc renoncer à cette complicité méthaphysique (...) sans risquer d'effacer la différence dans l'identité à soi d'un signifié réduisant en soi son signifiant60".

55. Voir par exemple leur examen des procédures intertextuelles ou ce qu'ils dénomment le "secondary system referenciation which becomes operative when a text thrusts outward to other texts", dans le "TRG Report 2, Narrative (Part 5)", "The Search for Nonnarrative Prose" (Part 2), Open Letter, 2e série, no 9, automne 1974, p. 52. Le champ théorique se situe ici dans le contexte kristevien et derridien du problème. Voir spécifiquement Julia Kristeva, Recherches pour une sémanalyse (extraits), Paris, Seuil, 1969, p. 18, 54, 91, 103-105, 107, 130-135, 245-254, 271-273 et récemment. Gérard Genette, Palimpsestes, Paris, Seuil, 1982.

56. Julia Kristeva, Recherches pour une sémanalyse (extraits), op. cit., p. 54, en français dans le texte.

57. Les termes choisis par le TRG sont "semantic cipher", voir"The Politics of the Referent," op. cit., p. 64.

58. Ibid., p. 64.

59. L'Écriture et la Différence, Paris, Seuil, 1967, p. 412.

60. Ibid., p. 413. 
Cette violence contre l'ontologie du signe, qui a tant préoccupé linguistes et philosophes, s'avère être ici un choix politique. Pour Steve McCaffery la déconstruction du signe (ou dans les termes de Derrida sa réduction à un concept métaphysique) implique aussi une déconstruction du mot, de la phrase " of the whole functioning of a linearized, serialized capitalist society61". Le sa n'a pas de sé, n'est pas obligé de signifier quoi que ce soit62. Le lecteur est celui qui réintroduit un contenu dans le contenant. Abandonné à lui-même dans un univers sémantique dépourvu d'absolus, il se découvre, producteur de sens; les vieilles dichotomies s'écroulent et lire équivaut à écrire. Mais ce réaménagement des rôles pratiqué par le TRG introduit un autre concept du texte, qui celui-là n'a rien à voir avec le système d'analyse barthien et post-structuraliste, et qu'on pourrait appeler la lecture comme acte de géomancie. La tâche du lecteur consiste alors à réorganiser les mouvements d'énergie présents, perçus dans le texte63. Ce passage d'un mode interprétatif contemporain à l'herméneutique d'un univers primitif et magique surprendra sans doute les lecteurs du TRG, peu habitués à une perspective mystique du langage (le mot est énergie et "carrier of healing processes64"). Nichol se réfère ici à une légende amérindienne (comme du reste, à leur façon, bill bissett et Daphne Marlatt), celle de Palongahoya qui ouvrait la bouche pour chanter la gloire du Créateur et dont l'axe vibratoire corporel vibrait en harmonie avec celui du cosmos. Mais arriva le Corbeau qui trompa les humains et ces derniers commencèrent à utiliser le langage différemment, avec masques, codes et mensonges. Et la légende dit que ceci se passa quatre fois 65 .

63. TRG Report 2 : Narrative (Part 3), "Charting the Obvious", Open Letter, 3e série, no 9, automne 1978, p. 67.

64. "bp Nichol/Interview", Capilano Review, op. cit., p. 325.

65. "The importance of sound poetry, for me, at that time, was to free the emotional content of speech from ideation or from words, necessarily, and to just be able to let out the voice. And then that once the voice had been let out then the words would follow. I always go back to that Palongahoya legend (...) that necessity to not use words as a masking". Ibid.

61. Steve McCaffery, "Absent Pre-Sences", Open Letter, 4e série, no 9, automne 1978, p. 130.

62. Ibid., p. 131. McCaffery se réfère ici Au plaisir du texte de Roland Barthes. 
Des Amérindiens en particulier et de la science de la géomancie, le TRG a retenu cette perception du langage paysage magique, du langage énergie magique. On pourrait aisément parler de croisement, d'hybridité théorique au sujet du TRG. Et effectivement le vécu qui fut le leur à Toronto dans les années 70 et 80 les porterait à la fois vers le post-structuralisme (cette intelligentsia européenne qui a connu une réception importante au USA) et vers les valeurs thérapeutiques et communautaires qui ont constitué un des fondements de leur expérience quotidienne66. Le concept d'énergie en particulier, si présent chez les post-modernistes de la Côte ouest, trouve dans le TRG une inflexion particulière, liée à la pratique de la poésie sonore, à sa performance et à l'utilisation collective qui peut en être faite. Deux groupes de poètes phoniques gravitant autour du TRG sont les Horsemen et les Owen Sound68. A leurs yeux de telles performances actualisent la mise en scène d'une certaine dynamique subsconsciente. Le premier terme appartenant au poète, le deuxième au public. La structure de cet échange est communautaire. McCaffery la dénomme "écouture69". Cette définition n'est pas la propriété exclusive de la poésie phonique. Pour n'importe quel postmoderniste, performance ou texte pourraient être "a configuration of discharges, graphic or semantic, arising from a reader's engagement with a text70". Il faut se hâter d'ajouter que la poésie sonore a illustré parfaitement le développement et la mise en ouvre d'un tel concept ${ }^{71}$.

Il semblerait, en définitive, que ce que vise la politique de la "référentialité" (pour emprunter l'expression de McCaffery) serait moins un signe évacué que le moi humain, lavé, purifié, libéré de ses terreurs, de ses passions, en somme un moi guéri : "a healing, the act of seeing and the being free from what one has seen 72 ",

66. Nichol et McCaffery faisaient partie de Therafields, communauté qui pratiquait la thérapie de groupe. Ils avaient également eu des contacts fréquents avec Jerome Rothenberg, poète phonique américain qui avait fait des recherches anthropologiques sur la musique et le sacré dans les cultures primitives. Voir "Préface: primitive means complex" in Aesthetics contemporary, ed. Richard Kostelanetz, Buffalo, Prometheus Books, 1978, p. 337-380. Voir aussi du même auteur Technicians of the Sacred, New York, Doubleday, 1968.

67. "The Four Horsemen" est un groupe de poètes phoniques fondé en 1970 et composé de Rafael Barretto Rivera, Paul Dutton, Steve McCaffery et bp Nichol. Ce dernier se retira après quelques années et les 'Four Horsemen' devinrent les "Horsemen".

68. "Owen Sound" groupe de poètes phoniques associés à Therafields, commença à produire en 1975. Son nom est emprunté à une petite ville située au nord de Toronto, célèbre pour la pureté de son eau et sa fraîcheur virgilienne.

69. McCaffery, "Discussion, Genesis, Continuity", Sound Poetry Catalogue, Toronto, Underwhich ed. 1979, p. 32-33.

70. "Charting the obvious", Opus cit. p. 65.

71. Les Dadaisstes, en particulier Hugo Ball, Tristan Tzara et Karl Schwilters, ont fréquemment et amplement fait référence à ces problèmes. Voir Dada spectrum: The Dialectics of Revolt, ed. Stephen Foster \& Rudolf Kuenzli, Madison, Coda Press, 1979.

72. Sound Poetry Catalog, p. 33. 
Mais les Horsemen accorderont à la performance une valeur dont le texte purement visuel sera malheureusement dépourvu. C'est ce qu'Antonin Artaud appelait non pas "la désacralisation des rêves mais l'utilisation de la parole dans un sens concret, spatial, objet solide qui ébranle les choses... idée élémentaire, magique, reprise par la psychanalyse moderne 73 ", et plus loin: " $j$ 'essaie de rendre sa vieille efficacité magique, son efficacité envoûtante, intégrale au langage de la parole dont on a oublié les mystérieuses possibilités $74 "$.

Il apparaît vingt ans plus tard que ce qui aura fait la réputation de Nichol, ce sont ses explorations visuelles, ses poèmes objets, plus que les réflexions du TRG sur la théorie et la langue entre 1975 et 1984. En fait les commentaires suscités à l'occasion de la remise du Prix du Gouverneur Général en 1971 de même que les réactions outrées des critiques au sujet de ses textes enveloppés dans des boîtes, enrubannés ou enroulés sous formes de mystérieux manuscrits lui auront valu une notoriété un peu artificielle75, semblable à celle que la pop-poetry et l'avant-garde se sont vu réserver de tout temps dans diverses cultures.

La parution de Martyrology, poème narratif 76 commencé en 1972 et dont les volumes se succèdent, a changé la nature et l'attitude de son public. Il cesse d'être le poète des désopilantes clowneries déployées dans Cronk et Ganglia (les deux petites revues ronéotypées et produites par lui dans les années 60 , à l'époque où les concrétistes français, allemands et brésiliens se dispersaient à coeur joie sur ces feuilles de papier à l'encre épaisse et poisseuse). Avec la Martyrology il insère dans le champ de la textualité la vie des saints et du langage, métaphysique et mythologie conjointes face à la mort du Logos et du Père.

73. Antonin Artaud, le Théâtre de la cruauté cité par Derrida dans "La clôture de la représentation", l'Écriture et la Différence, p. 350.

74. Ibid., p. 353.

75. Voir par exemple le rapport Hansard, 10 juin 1971, p. 6554-6557; 29 juin 1971, p. 7458. Les commentaires du député de Lambton-Kent, Mac T. McCutcheon, sont particulièrement savoureux : "in view of the recent Canada Council grant based on the writing of a treatise relating to the life of Billy the Kid, can the parliamentary secretary say if this is a change of direction from subsidizing Marxism to markmanship with short guns?" Pornographique, ordurier, grossier, furent quelques autres des épithètes utilisées par les députés de la Chambre à Ottawa ce jour-là.

76. "Nichol is working within an open-ended tradition of the long poem, begun in this century by Pound's Cantos, and The Martyrology is perhaps the most important epic ever written in Canada." Le jugement de Stephen Scolie est cité par Douglas Barbour dans "bp Nichol: The Life and the Letters of Life", Essays in Canadian Writing, no 9, hiver 1977-1978, p. 97. 
Depuis que ces deux derniers nous ont été arrachés, il ne nous reste qu'un succédané de langue : "a prototype/perfect model of the robot run amuck, a dead speech fr we have lost the gift of tongues 77 '". Nos mots sont notre seul et dernier espoir, ce sont les saints qui vont tenter de nous amener vers la Rédemption. Ils constituent un intermédiaire entre le quasiinaccessible Logos et nous-mêmes. "They have left heaven and though they never felt at home upon the earth they have come to help us reclaim our heritage, the mythology which founded our existence 78 '. C'est une expérience que Nichol décrit comme collective, nous sommes tous liés et engagés, car le langage à travers les langues nous enchaîne les uns aux autres et la rédemption sera nécessairement un processus collectif, une catharsis comparable à "l'écouture"79. Les saints interviendront en notre faveur "to bring out the cleasing of speech and the end to our private hells80".

L'impact le plus important de ce poème infini surgit de son fusionnement de deux traditions : la tradition grecque platonicienne, le Père-Logos à jamais inaccessible au commun des mortels81 et la métaphysique amérindienne de Palongahoya. Le narrateur cherche à retrouver l'harmonie cosmologique perdue. Ainsi commence la longue quête de ce Dieu-son:

how can $\mathrm{i}$ live without you

only the skies empty my tears

hell $i$ could fill the space with moaning

of you are gone \& i am left

lonely father 82

Pourtant la situation n'est pas irrémédiable. Saints et poètes peuvent essayer de retrouver l'énergie primordiale de la parole :

the language destroyed made whole again 83

we must protect the sacred energy84

77. Book I, The Martyrology, Toronto, Coach House, 1976, sans pagination.

78. Book III, op. cit., sans pagination.

79. Voir McCaffery là-dessus, Sound Poetry Catalog, opus cit. p. 33 et aussi Paul Dutton "Open Journal Milwaukee", Open Letter, 3e série, no 8, printemps 1978, p. 44-51.

80. Book $I I I$, sans pagination.

81. Voir Derrida, "La pharmacie de Platon", la Dissémination, Paris, Seuil, 1972, p. 90-95.

82. Book $I \& I I$, sans pagination.

83. Ibid.

84. Ibid. 
Le texte dans son ensemble se présente comme une quête phénoménologique du Logos à travers le langage, voyage-découverte au delà du "lung wage" ou plutôt à partir de celui-ci, avec l'intercession des saints auxquels Nichol se réfère ainsi :

you have no name now
only a being so alive
i know you're all still with me
linked as one
energy moving into song 85

Mais ce voyage n'est pas purement individuel, la rédemption désirée est un processus collectif. Par exemple, dans la huitième partie du livre III le poète établit le lien entre le "nous" de sa communauté présente (un groupe qui bâtit une maison) et les migrations historiques de l'humanité vers le Nord telles qu'elles sont reflétées dans le développement des langues indoeuropéennes. Cette complexe histoire linguistique est examinée ici avec de multiples références aux déplacements des lettres à l'intérieur des mots. "It's an issue of names," dit Nichol et l'espoir final est contenu dans l'énoncé suivant:

who pursues his father to the end finds him again tribal unity

restores the we

regains that sense of what his place was not

to praise falsely who should not be praised

but to give him due

the one who was before you \& fathered you 86

Il serait intéressant de comparer la perte du Père, la mort du logos chez les post-modernistes canadiens et québécois. Le désespoir de Nichol trouve son antithèse la plus symétriquement parfaite dans la poésie-célébration de François Charron (mais d'autres exemples pourraient être cités là-dessus; Nicole Brossard, Yolande Villemaire, Claude Beausoleil illustreraient de manière tout aussi convaincante cette perte-découverte). Charron envisage la modernité comme une libération à l'égard de la vieille théologie du sens, du monothésime absolu, comme une libération de/par la matière87: "il y a le signifiant incompréhensible (...) il y a l'incommunicable beauté de la matière qui chante et qui pense88". "La nouvelle écriture désamorce le rapport où le fils se croit engendré par le Père89".

85. Book III, sans pagination.

86. Book III. L'obsession du Logos-Père dans Martyrology ne devrait pas être interprétée comme un rejet de la féminité. Voir en particulier dans ce même livre la référence à sa découverte "in the twin mouths of women of W's omen".

87. François Charron, "L'Écriture commence par un rêve", la Nouvelle Barre du jour, no. 90-91, mai 1980, p. 25.

88. Ibid., p. 25.

89. Ibid., p. 22 
Les poètes canadiens et les poètes québécois attaquent avec une égale détermination le clair et le lisible, la déconstruction est leur priorité explicite. La différence - et elle est capitale - est que pour les anglophones il y a une énergie à rejoindre, exprimée dans la matière mais également ancrée dans un ailleurs (Palongahoya, le Logos, le Père) spirituel, parfait, accessible. Pour les Québécois de la modernité (Nouvelle Barre du jour et Herbes rouges) le salut est dans la matière et, si rédemption il y a, elle a tout à voir avec le rejet du théologique, du lisible et du Père: "vous êtes en train de vous réveiller, dans la grande nuit patriarcale et monothéiste (...) vous vous mettez à barbouiller, à griffonner, à danser, à fredonner 90 ". Il serait intéressant - mais ce n'est ni le moment, ni le lieu pour le faire - d'analyser les différentes idéologies à l'ouuvre dans ces deux post-modernismes et d'essayer d'établir un réseau topologique et paradramatique dans le cadre de cette même problématique.

Il n'en demeure pas moins que ce bref parcours dans le champ de la poétique canadienne depuis vingt ans nous permet de dégager quelques lignes de force. Déconstruire, bouleverser l'ordre politico-linguistique est une nécessité pour le post-modernisme canadien. Mais au delà de ces pulsions et de ces impératifs, les poètes essayent de rejoindre un autre ordre, primordial, pré-occidental, méta-occidental et méta-physique serait-on tenté de dire. Ce bond à travers le temps et le langage manifeste l'attente d'une certaine révélation, une foi en certaine épiphanie (Joyce encore) qui les éloigne tout autant de leurs compagnons chercheurs québécois que des Américains. Le post-modernisme au Canada suit un chemin qui lui est propre.

90. François Charron, "Notes sur l'expérience de la peinture ", ies Herbes rouges, nos 75-76, novembre 1979, p. 18. Voir aussi de Normand de Bellefeuille "Le caca et le lisible", les Herbes rouges, no 38 , août 1976, p. 1-27. 